\title{
Laparoscopic radical prostatectomy and resection of rectum performed together: first experience
}

\author{
Milos Brodak ${ }^{1}$, Julius Orhalmi ${ }^{2}$, Josef Kosina ${ }^{1}$, Michal Balik $^{1}$, Jaroslav Pacovsky ${ }^{1}$ \\ ${ }^{1}$ Department of Urology, Charles University Hospital, Hradec Kralove, Czech Republic \\ ${ }^{2}$ Department of Surgery, Charles University Hospital, Hradec Kralove, Czech Republic
}

Videosurgery Miniinv 2015; 10 (3): 442-444

DOI: $10.5114 /$ wiitm.2015.54054

\begin{abstract}
Introduction: Laparoscopy is an increasingly used approach in the surgical treatment of rectal cancer and prostate cancer. The anatomical proximity of the two organs is the main reason to consider performing both procedures simultaneously.

Aim: To present our first experience of laparoscopic rectal resection and radical prostatectomy, performed simultaneously, in 3 patients.

Material and methods: The first patient was diagnosed with locally advanced rectal cancer and tumor infiltration of the prostate and seminal vesicles. The other 2 patients were diagnosed with tumor duplicity. The surgery of the first patient started with laparoscopic prostatectomy except division of the prostate from the rectal wall. The next step was resection of the rectum, extralevator amputation of the rectum and vesicourethral anastomosis. In the other patients, resection of the rectum, followed by radical prostatectomy, was performed.

Results: The median follow-up was 12 months. The median operation time was $4 \mathrm{~h} 40 \mathrm{~min}$, with blood loss of $300 \mathrm{ml}$. The operations and postoperative course were without incident in the case of 2 patients. However, 1 patient had stercoral peritonitis and a vesicorectal fistula in the early postoperative stage. Sigmoidostomy and postponed ureteroileal conduit were carried out. All patients were in oncologic remission.

Conclusions: Combined laparoscopic rectal resection and radical prostatectomy is a viable option for selected patients with locally advanced rectal cancer or tumor duplication. The procedures were completed without complications in 2 out of 3 patients.
\end{abstract}

Key words: laparoscopic radical prostatectomy, laparoscopic resection of rectal cancer, stercoral peritonitis.

\section{Introduction}

The incidence rate of rectal and prostate cancer is high and growing, and laparoscopic resection of the rectum and radical prostatectomy are carried out increasingly [1-3]. The anatomical proximity of these two organs was the main reason for performing both procedures simultaneously in case of locally advanced rectal tumor or tumor duplicity. Combined surgery could be beneficial for patients, because they underwent only one operation. Moreover, the laparoscopic approach is associated with reduction of invasiveness and allows a faster recovery. On the other hand, a combined procedure increases the risk of complications and requires cooperation of surgical and urological teams.

\section{Aim}

The aim of this paper is to present initial experiences of combined laparoscopic surgery as radical prostatectomy and resection of the rectum. The au-

\section{Address for correspondence}

Milos Brodak MD, PhD, Department of Urology, Charles University Hospital, 581 Sokolska St, 50005 Hradec Kralove, Czech Republic, phone: +42 0495833378, fax:+42 0495833431, e-mail: brodak@fnhk.cz 
thors evaluated the results from the procedures as well as complications.

\section{Material and methods}

The evaluation involved 3 patients who underwent laparoscopic radical prostatectomy and rectal resection. The median age was 59 years. The first patient was diagnosed with locally advanced rectal cancer and infiltration of the prostate and seminal vesicles. The other two patients were diagnosed with tumor duplicity, prostate and rectal cancer. All 3 patients underwent neoadjuvant and adjuvant radiotherapy for rectal cancer. Prior to the surgery they were fully informed about the combined procedure, the risks of any complications and the possibilities of alternative approaches.

The first patient was a 47-year-old man, with locally advanced rectal cancer and enlarged pelvic lymph nodes, but without distal metastases. The patient underwent laparoscopic combined surgery aimed at completely removing the tumor. The first phase of the surgery was radical prostatectomy. Full removal of the prostate was carried out, except division of the dorsal part of the prostate and seminal vesicles from the rectum. The prostate was cut off from the bladder and urethra. The next step was laparoscopic resection of the rectum, pelvic lymphadenectomy and sigmoidostomy. Subsequently extralevator amputation of the rectum and vesicourethral anastomosis was performed using the same approach. After recovery from the surgery the patient underwent adjuvant chemotherapy and radiotherapy. The definitive stage of the tumor was ypT3ypN1b (16/3) pMO, RO and tumor grading III.

The next 2 patients were men with tumor duplicity, aged 59 and 73 . Both cancers were local, with no lymphadenopathy or distal metastases. The primary disorder was rectal cancer, and prostate cancers were found during the process of tumor staging. The first surgical step was the resection of the rectum and intestinal anastomosis. The following step was radical prostatectomy with vesicourethral anastomosis. The radical prostatectomy was performed by a urologic team and rectal resection by an abdominal surgical team. An indwelling urinary catheter was left for 14 days after surgery. Cystography was performed before removing the catheter. The surgical results and complications were assessed.

\section{Results}

The median follow-up was 12 months. The median operation time was $4 \mathrm{~h} 40$ min with blood loss of $300 \mathrm{ml}$. The operations on all three patients were performed without complications. The first two patients had an uncomplicated postoperative course. The third patient had serious complications in the early postoperative period. Stercoral peritonitis was diagnosed during the first postoperative day. The patient underwent two urgent reoperations. Colostomy and repeated cleaning of the peritoneal cavity with temporary drainage were performed. An additional complication was a vesicorectal fistula. It was found through urine secretion to drain during the fifth postoperative day. The patient had a small fistula in the vesicourethral anastomosis proven on cystography and by flexible cystoscopy. An indwelling urinary catheter was used as the initial transitional treatment. The next treatment was the York-Mason procedure, which occurred 6 months after the laparoscopic surgery. This procedure was unsuccessful, with recurrence of a fistula. The last procedure was urine diversion, and the ureteroileal conduit was performed. All patients were in complete oncologic remission. The first patient with locally advanced rectal cancer was urine and stool continent. The second patient suffered mild urinary incontinence and was stool continent. The urine incontinence tended to improve, and the patient needed only one small incontinence pad per day. The third patient had a colostomy and ileal conduit.

\section{Discussion}

The idea of combined surgery is based on proximity of the prostate and rectum in the male pelvis. The initial idea to carry out this procedure originated while planning the optimal treatment of a young patient diagnosed with locally advanced rectal cancer. The main problem of this particular patient was tumor infiltration of the prostate and seminal vesicles. The main objective was radical surgery with total resection of the tumor with no positive surgical margin or residual tumor. Previous positive experience with the laparoscopic resection of the rectum as well as radical prostatectomy was the main reason we decided in favor of the laparoscopic combined approach. The surgery was planned to involve radical prostatectomy with release of the prostate from the bladder, urethra and lateral pelvic floor as the first step. After this procedure, the prostate and seminal 
vesicles remained on the rectum, and the resection of the rectum with tumor was complete, with no positive surgical margin. The pelvic lymphadenectomy was carried out with the same laparoscopic approach. The rectum with the prostate was removed via extralevator resection [4]. The same approach was used for vesicourethral anastomosis, with an outstanding overview. The excellent result of the surgery consequently led to the planning and execution of this approach on the other 2 patients.

The second surgery proceeded as intended without complications. The intestinal continuity was performed via laparoscopic anastomosis. Positive results encouraged continuing the exact practice in the third surgery. This surgery however was associated with substantial complications. The first complication was stercoral peritonitis with leakage of stool from the intestinal anastomosis. The treatment was to reoperate with sigmoidostomy. A further complication was urine leakage to the rectal stump through a fistula in the vesicourethral anastomosis.

The indwelling of a urinary catheter was temporary treatment. The only risk factor of this patient was his higher age in comparison with the other 2 patients. He was 73 years old.

The extensive experience in laparoscopic surgery was the first condition for carrying out this difficult procedure. Laparoscopic radical prostatectomy, purely laparoscopic or robot-assisted, has become the most widely used surgical method in many developed countries [5]. One of the main advantages is in better a visual overview due to the use of video transmission and high magnification [6]. The two procedures have some similarities, especially in separating organs from the pelvic floor with similar placement of laparoscopic ports. These similarities were taken into consideration in our combined procedures. The next important advantage of the laparoscopic approach is the low blood loss, which was confirmed during all three operations.

The radiotherapy used in management of rectal tumors was a risk factor in the healing process. Salvage radical prostatectomy carried out for recurrence following radiotherapy of prostate cancer is commonly associated with higher risk of poor healing or complications.

This type of surgery is demanding and with significant risk of complications $[7,8]$. It is reserved only for a limited number of patients with high motivation. Vast experience with laparoscopic surgery and excellent abdominal surgical and urological collaboration are a necessity. The main limitation of this work is the small number of patients. According to the authors' knowledge, there are no existing publications reporting this type of combined surgery.

\section{Conclusions}

Combined laparoscopic surgery with radical prostatectomy and resection of the rectum, carried out simultaneously, is a feasible procedure in cases of locally advanced rectal cancer or tumor duplicity. The main advantage of this procedure is the reduction of invasiveness. Disadvantages are the more complex procedure and the risk of complications. This procedure should be limited to centers with extensive experience with laparoscopy.

\section{Acknowledgments}

The article was supported by program PRVOUK P37/04.

\section{Conflict of interest}

The authors declare no conflict of interest.

\section{References}

1. Wirth MD, Brandt HM, Dolinger $\mathrm{H}$, et al. Examining connections between screening for breast, cervical and prostate cancer and colorectal cancer screening. Colorectal Cancer 2014; 3: 253-63.

2. Müller S, Grønning LE, Nilsen FS, Mygland V. Robotic and minimal access surgery: technology and surgical outcomes of radicalprostatectomy for prostate cancer. Expert Rev Anticancer Ther 2014; 30: 1-5.

3. van der Pas MH, Haglind E, Cuesta MA, et al. Laparoscopic versus open surgery for rectal cancer (COLOR II): short-term outcomes of a randomised, phase 3 trial. Lancet Oncology 2013; 14: 210-8.

4. Orhalmi J, Klos K, Jackanin S, Holéczy P. Intersphincteric resection of the rectum. Rozhl Chir 2012; 91: 101-4.

5. Hu JC, Gandaglia G, Karakiewicz PI, et al. Comparative effectiveness of robot-assisted versus open radical prostatectomy cancer control. Eur Urol 2014; 66: 666-72.

6. Golabek T, Jarecki P, Jaskulski J, et al. Modified technique for laparoscopic running vesicourethral anastomosis. Videosurgery Miniinv 2014; 9: 357-61.

7. Skrovina M, Soumarova R, Kycina R, et al. Anastomotic leakage after laparoscopic total mesorectal excision for low rectal cancer. Videosurgery Miniinv 2011; 6: 5-11.

8. Soares R, Di Benedetto A, Dovey Z, et al. Minimum five-year follow-up of 1,138 consecutive laparoscopic radical prostatectomies. BJU Int 2015; 115: 546-53.

Received: 12.04.2015, accepted: 19.08.2015. 